\title{
EDITORIAL
}

\section{Is respiratory controller arrhythmia an artifact or a real phenomenon?}

\author{
A. Jubran
}

Disturbances of the respiratory control system are commonly suspected in patients with chronic obstructive pulmonary disease (COPD) experiencing acute respiratory failure. Studies evaluating the mechanisms responsible for these disturbances have not yielded a uniform answer, perhaps because most research has primarily focused on the mean response of the respiratory controller to various perturbations. However, when evaluating the performance of a control system, it is important to also take into account the ability of the respiratory control system to maintain or vary its response over time [1].

Some insight into controller variability can be gained by measuring the coefficients of variation, i.e. standard deviation divided by the mean, of breath components. Studies have revealed that healthy subjects display a wide range of breath-to-breath variability of the primary breath components with older subjects displaying a more variable pattern than younger ones $[2,3]$. In addition, chemical stimulation and mechanical loading have been shown to alter the variability of breathing in a manner that is specific to the nature of the stimulus or load [4-6].

Patients with COPD appear to display less variability in tidal volume $(V \mathrm{~T})$ and respiratory frequency than normal subjects [7]. Moreover, episodes of crescendo-decrescendo changes in $V \mathrm{~T}$ and oscillations in end-expiratory lung volume analogous to Cheyne-Stokes breathing have been reported in COPD patients [8]. An association between hypercapnia and increased variability has been suggested in this patient group [9]. Thus, an increase in the variability of breathing may itself be a manifestation of abnormal control of breathing in patients with COPD.

In this issue of the Journal, VITACCA et al. [10] report on breathing pattern during spontaneous breathing based on measurements from both oesophageal pressure and flow tracings in 34 patients with COPD recovering from acute exacerbation. Fifty-nine per cent of the patients had oesophageal pressure swings during expiration, which the investigators referred to as "premature inspirations". They divided the patients into two subgroups depending on the presence or absence of these "premature inspirations". While the mean values of physiological variables were not different between the two groups, coefficients of variation of intrinsic positive end-expiratory pressure, work of breathing and breathing pattern were increased in the group of patients with "premature inspirations". This study highlights the importance of examining the breath-to-breath

Correspondence: A. Jubran, Division of Pulmonary and Critical Care Medicine, Edward Hines Jr, Veterans Affairs Hospital, Route 111N Hines, IL 60141, USA. Fax: 17085317907. variability of respiratory controlled output when studying the control of breathing.

Caution should be exercised when interpreting the findings of this study by VITACCA et al. [10] since coefficients of variation were calculated on the basis of only eight breaths. Because of the considerable breath-to-breath variability in humans, ventilation should be monitored over a prolonged period of time and calculations should be based on a large number of breaths rather than selecting a handful of "representative" breaths [2]. Moreover, the use of a mouthpiece in $50 \%$ of the patients of VITACCA et al. [10] to measure breathing pattern is problematic since the instrumentation itself can alter respiratory centre output $[11,12]$.

The assumption by VITACCA et al. [10] that oesophageal pressure swings during expiratory flow represents a "premature" inspiratory effort is not easily accepted without concomitant recordings from other muscle groups (including abdominal muscles). If, indeed, the oesophageal pressure swings signal inspiratory events, several factors may have contributed to their origin. Firstly, these efforts could represent an increase in the natural variability of the controller in terms of the magnitude of respiratory effort and timing on a breath-to-breath basis [13]. Secondly, the oesophageal pressure swings could indicate a decrease in the effectiveness of inspiratory pressure generation, due to dynamic hyperinflation with consequent inspiratory muscle shortening. The associated increase in elastic recoil pressure will also act as a threshold load, and, thus, markedly increase the magnitude of a patient's total effort required to start inspiration. Indeed, an increase in elastic recoil pressure is an important reason why critically-ill patients have difficulty in triggering a mechanical ventilator [14]. Thirdly, a larger $V \mathrm{~T}$ could be responsible for the appearance of oesophageal pressure swings. The larger the $V \mathrm{~T}$, the longer will be the time needed for exhalation. After a "premature inspiration", extra time will be available for lung volume to return to relaxed volume, increasing the likelihood of effective efforts. Likewise, a larger $V_{\mathrm{T}}$ can activate the Hering-Breuer reflex and thus decrease neural inspiratory time and inspiratory effort [15]. Such a mechanism is suggested by figure 1 in VITACCA et al. [10], where a "premature inspiration" appears to be preceded by a sigh.

The clinical significance of these "premature inspirations" remains uncertain. In a subgroup analysis of patients who were on long-term oxygen therapy, VITACCA et al. [10] observed higher inspiratory load and weaker inspiratory muscles in patients with "premature inspirations". These data suggest that these events may be a marker of severity of disease in patients with COPD. Further studies are needed to clarify this issue. 
In summary, VITACCA et al. [10] are to be congratulated for heightening our awareness of the variability of inspiratory efforts during spontaneous breathing in patients with chronic obstructive pulmonary disease. Future studies evaluating this compelling issue should include direct measurements of the neural and mechanical properties of the respiratory system to define the true origin of oesophageal pressure swings. Moreover, these parameters should be monitored over prolonged periods of time in order to assess the ability of the controller to vary its output over time. Sophisticated signal analysis is now available which in conjunction with breathing pattern analysis could prove to be very powerful in our understanding of the mechanisms of respiratory control in patients with chronic obstructive pulmonary disease.

\section{References}

1. Cherniack NS, Longobardo GS. Abnormalities in respiratory rhythm. In: Cherniack NS, Widdicombe JG, eds. Handbook of Physiology. Bethesda, American Physiology Society, 1986; pp. 729-749.

2. Tobin MJ, Mador MJ, Guenther SM, Lodato RG, Sackner MA. Variability of resting respiratory drive and timing in healthy subjects. J Appl Physiol 1988; 65: 309-331.

3. Tobin MJ, Yang KL, Jubran A, Lodato RF. Interrelationship of breath components in neighboring breaths of normal eucapnic subjects. Am J Respir Crit Care Med 1995; 152: 1967-1976.

4. Jubran A, Grant BJB, Tobin MJ. Effect of hyperoxic hypercapnia on variational activity of breathing. $\mathrm{Am} \mathrm{J}$ Respir Crit Care Med 1997; 156: 1129-1139.

5. Brack T, Jubran A, Tobin MJ. Effect of elastic loading on variational activity of breathing. Am J Respir Crit Care Med 1997; 155: 1341-1348.

6. Brack T, Jubran A, Tobin MJ. Effect of resistive loading on variational activity of breathing. Am J Respir Crit Care Med 1998; 157: 1756-1763.

7. Loveridge B, West P, Anthonisen NR, Kryger MH. Breathing patterns in breathing with chronic obstructive pulmonary disease. Am Rev Respir Dis 1984; 130: 730733.

8. Tobin MJ, Chadha TS, Jenouri G, Birch SJ, Gazeroglu HB, Sackner MA. Breathing patterns: II: diseased subjects. Chest 1983; 84: 286-294.

9. Wolkove N, Altose MD, Kelsen SG, Cherniack NS. Respiratory control abnormalities in alveolar hypoventilation. Am Rev Respir Dis 1980; 122: 163-167.

10. Vitacca M, Porta R, Bianchi L, Clini E, Ambrosino N. Differences in spontaneous breathing pattern and mechanics in severe COPD patients recovering from acute exacerbation. Eur Respir J 1999; 13: 365-370.

11. Perez W, Tobin WJ. Separation of factors responsible for change in breathing pattern induced by instrumentation. $J$ Appl Physiol 1985; 59: 1515-1520.

12. Gilbert R, Auchincloss JH, Brodsky J, Broden W. Changes in tidal volume, frequency, and ventilation induced by their measurement. J Appl Physiol 1972; 33: 252-254.

13. Parthasarathy S, Jubran A, Tobin MJ. Cycling of inspiratory and expiratory muscle groups with the ventilator in airflow limitation. Am J Respir Crit Care Med 1998; 158: 1471-1478.

14. Leung P, Jubran A, Tobin MJ. Comparison of assisted ventilator modes on triggering, patient effort and dyspnea. Am J Respir Crit Care Med 1997; 155: 1940-1948.

15. Clark FJ, von Euler C. On the regulation of depth and rate of breathing. J Physiol (Lond) 1972; 222: 267-295. 